\title{
Inverse Coefficient Problem of the Parabolic Equation with Periodic Boundary and Integral Overdetermination Conditions
}

\author{
Fatma Kanca \\ Department of Management Information Systems, Kadir Has University, 34083 Istanbul, Turkey \\ Correspondence should be addressed to Fatma Kanca; fatma.kanca@khas.edu.tr
}

Received 6 May 2013; Accepted 23 August 2013

Academic Editor: Daniel C. Biles

Copyright (C) 2013 Fatma Kanca. This is an open access article distributed under the Creative Commons Attribution License, which permits unrestricted use, distribution, and reproduction in any medium, provided the original work is properly cited.

This paper investigates the inverse problem of finding a time-dependent diffusion coefficient in a parabolic equation with the periodic boundary and integral overdetermination conditions. Under some assumption on the data, the existence, uniqueness, and continuous dependence on the data of the solution are shown by using the generalized Fourier method. The accuracy and computational efficiency of the proposed method are verified with the help of the numerical examples.

\section{Introduction}

Denote the domain $D_{T}$ by

$$
D_{T}=\{(x, t): 0<x<1,0<t \leq T\} .
$$

Consider the equation

$$
u_{t}=a(t) u_{x x}+F(x, t)
$$

with the initial condition

$$
u(x, 0)=\varphi(x), \quad 0 \leq x \leq 1,
$$

the periodic boundary condition

$$
\begin{gathered}
u(0, t)=u(1, t), \quad u_{x}(0, t)=u_{x}(1, t), \\
0 \leq t \leq T,
\end{gathered}
$$

and the overdetermination condition

$$
\int_{0}^{1} x u(x, t) d x=E(t), \quad 0 \leq t \leq T .
$$

The problem of finding a pair $\{a(t), u(x, t)\}$ in (2)-(5) will be called an inverse problem.
Definition 1. The pair $\{a(t), u(x, t)\}$ from the class $C[0, T] \times$ $C^{2,1}\left(D_{T}\right) \cap C^{1,0}\left(\bar{D}_{T}\right)$ for which conditions (2)-(5) is satisfied and $a(t)>0$ on the interval $[0, T]$, is called a classical solution of the inverse problem (2)-(5).

The parameter identification in a parabolic differential equation from the data of integral overdetermination condition plays an important role in engineering and physics [1-7]. This integral condition in parabolic problems is also called heat moments [5].

Boundary value problems for parabolic equations in one or two local classical conditions are replaced by heat moments [8-13]. These kinds of conditions such as (5) arise from many important applications in heat transfer, thermoelasticity, control theory, life sciences, and so forth. For example, in heat propagation in a thin rod, the law of variation $E(t)$ of the total quantity of heat in the rod is given in [8]. In [12], a physicalmechanical interpretation of the integral conditions was also given.

Various statements of inverse problems on determination of thermal coefficient in one-dimensional heat equation were studied in $[4,5,7,14]$. In papers $[4,5,7]$, the time-dependent thermal coefficient is determined from the heat moment.

Boundary value problems and inverse problems for parabolic equations with periodic boundary conditions are investigated in $[15,16]$. 
In the present work, one heat moment is used with periodic boundary condition for the determination of thermal coefficient. The existence and uniqueness of the classical solution of the problem (2)-(5) is reduced to fixed point principles by applying the Fourier method.

This paper organized as follows. In Section 2, the existence and uniqueness of the solution of inverse problem (2)(5) are proved by using the Fourier method. In Section 3, the continuous dependence on the solution of the inverse problem is shown. In Section 4, the numerical procedure for the solution of the inverse problem using the Crank-Nicolson scheme combined with an iteration method is given. Finally, in Section 5, numerical experiments are presented and discussed.

\section{Existence and Uniqueness of the Solution of the Inverse Problem}

We have the following assumptions on the data of the problem (2)-(5).

$$
\begin{aligned}
& \left(A_{1}\right) E(t) \in C^{1}[0, T], E^{\prime}(t)>0, \text { for all } t \in[0, T] \\
& \left(A_{2}\right) \varphi(x) \in C^{4}[0,1] \\
& \text { (1) } \varphi(0)=\varphi(1), \varphi^{\prime}(0)=\varphi^{\prime}(1), \varphi^{\prime \prime}(0)=\varphi^{\prime \prime}(1) \\
& \int_{0}^{1} x \varphi(x) d x=E(0) \\
& \text { (2) } \varphi_{n} \geq 0, n=1,2, \ldots ;
\end{aligned}
$$

$\left(A_{3}\right) F(x, t) \in C\left(\bar{D}_{T}\right) ; F(x, t) \in C^{4}[0,1]$ for arbitrary fixed $t \in[0, T]$

$$
\begin{aligned}
& \text { (1) } F(0, t)=F(1, t), F_{x}(0, t)=F_{x}(1, t), F_{x x}(0, t)= \\
& F_{x x}(1, t) ;
\end{aligned}
$$$$
\text { (2) } F_{n}(t) \geq 0, n=1,2, \ldots \text {, }
$$

where $\varphi_{n}=\int_{0}^{1} \varphi(x) \sin (2 \pi n x) d x, F_{n}(t)=\int_{0}^{1} F(x, t) \sin (2 \pi n x) d x$, $n=0,1,2, \ldots$

Theorem 2. Let the assumptions $\left(A_{1}\right)-\left(A_{3}\right)$ be satisfied. Then the following statements are true.

(1) The inverse problem (2)-(5) has a solution in $D_{T}$.

(2) The solution of inverse problem (2)-(5) is unique in $D_{T_{0}}$, where the number $T_{0}\left(0<T_{0}<T\right)$ is determined by the data of the problem.

Proof. By applying the standard procedure of the Fourier method, we obtain the following representation for the solution of (2)-(4) for arbitrary $a(t) \in C[0, T]$ :

$$
\begin{aligned}
u(x, t)=\sum_{n=1}^{\infty} & {\left[\varphi_{n} e^{-(2 \pi n)^{2} \int_{0}^{t} a(s) d s}+\int_{0}^{t} F_{n}(\tau) e^{-(2 \pi n)^{2} \int_{\tau}^{t} a(s) d s} d \tau\right] } \\
& \times \sin (2 \pi n x) .
\end{aligned}
$$

The assumptions $\varphi(0)=\varphi(1), \varphi^{\prime}(0)=\varphi^{\prime}(1), F(0, t)=$ $F(1, t)$, and $F_{x}(0, t)=F_{x}(1, t)$ are consistent conditions for the representation (2) of the solution $u(x, t)$ to be valid. Furthermore, under the smoothness assumptions $\varphi(x) \in C^{4}[0,1]$,
$F(x, t) \in C\left(\bar{D}_{T}\right)$, and $F(x, t) \in C^{4}[0,1]$ for all $t \in[0, T]$, the series (6) and its $x$-partial derivative converge uniformly in $\bar{D}_{T}$ since their majorizing sums are absolutely convergent. Therefore, their sums $u(x, t)$ and $u_{x}(x, t)$ are continuous in $\bar{D}_{T}$. In addition, the $t$-partial derivative and the $x x$-secondorder partial derivative series are uniformly convergent for $t \geq \varepsilon>0$ ( $\varepsilon$ is an arbitrary positive number). Thus, $u(x, t) \in$ $C^{2,1}\left(D_{T}\right) \cap C^{1,0}\left(\bar{D}_{T}\right)$ and satisfies the conditions (2)-(4). In addition, $u_{t}(x, t)$ is continuous in $\bar{D}_{T}$ because the majorizing sum of $t$-partial derivative series is absolutely convergent under the condition $\varphi^{\prime \prime}(0)=\varphi^{\prime \prime}(1)$ and $f_{x x}(0, t)=f_{x x}(1, t)$ in $\bar{D}_{T}$. Equation (6) can be differentiated under the condition $\left(A_{1}\right)$ to obtain

$$
\int_{0}^{1} x u_{t}(x, t) d x=E^{\prime}(t)
$$

and this yields

$$
a(t)=P[a(t)]
$$

where

$$
\begin{aligned}
& P[a(t)] \\
& =\frac{E^{\prime}(t)+\sum_{n=1}^{\infty}(1 / 2 \pi n) F_{n}(t)}{\sum_{n=1}^{\infty} 2 \pi n\left(\varphi_{n} e^{-(2 \pi n)^{2} \int_{0}^{t} a(s) d s}+\int_{0}^{t} F_{n}(\tau) e^{-(2 \pi n)^{2} \int_{\tau}^{t} a(s) d s} d \tau\right)} .
\end{aligned}
$$

Denote

$$
\begin{gathered}
C_{0}=\min _{t \in[0, T]} E^{\prime}(t)+\min _{t \in[0, T]}\left(\sum_{n=1}^{\infty} \frac{1}{2 \pi n} F_{n}(t)\right), \\
C_{1}=\max _{t \in[0, T]} E^{\prime}(t)+\max _{t \in[0, T]}\left(\sum_{n=1}^{\infty} \frac{1}{2 \pi n} F_{n}(t)\right), \\
C_{2}=E^{\prime}(0), \quad C_{3}=\sum_{k=1}^{\infty} 2 \pi n\left(\varphi_{n}+\int_{0}^{T} F_{n}(\tau) d \tau\right) .
\end{gathered}
$$

Using the representation (8), the following estimate is true:

$$
0<\frac{C_{0}}{C_{3}} \leq a(t) \leq \frac{C_{1}}{C_{2}} .
$$

Introduce the set $M$ as

$$
M=\left\{a(t) \in C[0, T]: \frac{C_{0}}{C_{3}} \leq a(t) \leq \frac{C_{1}}{C_{2}}\right\} .
$$

It is easy to see that

$$
P: M \longrightarrow M
$$

Compactness of $P$ is verified by analogy to [7]. By virtue of Schauder's fixed-point theorem, we have a solution $a(t) \epsilon$ $C[0, T]$ of $(8)$.

Now let us show that there exists $D_{T_{0}}\left(0<T_{0} \leq T\right)$ for which the solution $(a, u)$ of the problem $(2)-(5)$ is unique in 
$D_{T_{0}}$. Suppose that $(b, v)$ is also a solution pair of the problem $(2)-(5)$. Then from the representations (6) and (8) of the solution, we have

$$
\begin{aligned}
& u(x, t)-v(x, t) \\
& =\sum_{n=1}^{\infty} \varphi_{n}\left(e^{-(2 \pi n)^{2} \int_{0}^{t} a(s) d s}-e^{-(2 \pi n)^{2} \int_{0}^{t} b(s) d s}\right) \sin 2 \pi n(x) \\
& +\sum_{n=1}^{\infty}\left(\int_{0}^{t} F_{n}(\tau)\left(e^{-(2 \pi n)^{2} \int_{\tau}^{t} a(s) d s}-e^{-(2 \pi n)^{2} \int_{\tau}^{t} b(s) d s}\right) d \tau\right) \\
& \times \sin 2 \pi n(x), \\
& a(t)-b(t)=P[a(t)]-P[b(t)],
\end{aligned}
$$

where

$$
\begin{aligned}
& P[a(t)]-P[b(t)] \\
& =\frac{E^{\prime}(t)+\sum_{n=1}^{\infty}(1 / 2 \pi n) F_{n}(t)}{\sum_{n=1}^{\infty} 2 \pi n\left(\varphi_{n} e^{-(2 \pi n)^{2} \int_{0}^{t} a(s) d s}+\int_{0}^{t} F_{n}(\tau) e^{-(2 \pi n)^{2} \int_{\tau}^{t} a(s) d s} d \tau\right)} \\
& -\frac{E^{\prime}(t)+\sum_{n=1}^{\infty}(1 / 2 \pi n) F_{n}(t)}{\sum_{n=1}^{\infty} 2 \pi n\left(\varphi_{n} e^{-(2 \pi n)^{2} \int_{0}^{t} b(s) d s}+\int_{0}^{t} F_{n}(\tau) e^{-(2 \pi n)^{2} \int_{\tau}^{t} b(s) d s} d \tau\right)} .
\end{aligned}
$$

The following estimate is true:

$$
\begin{aligned}
|P[a(t)]-P[b(t)]| & \\
\leq & \frac{\left(E^{\prime}(t)+\sum_{n=1}^{\infty}(1 / 2 \pi n) F_{n}(t)\right)}{C_{2}^{2}} \\
& \cdot\left(\sum_{n=1}^{\infty} 2 \pi n \varphi_{n}\left(e^{-(2 \pi n)^{2} \int_{0}^{t} a(s) d s}-e^{-(2 \pi n)^{2} \int_{0}^{t} b(s) d s}\right)+\sum_{n=1}^{\infty} 2 \pi n\right. \\
& \left.\times\left(\int_{0}^{t} F_{n}(\tau)\left(e^{-(2 \pi n)^{2} \int_{\tau}^{t} a(s) d s}-e^{-(2 \pi n)^{2} \int_{\tau}^{t} b(s) d s}\right) d \tau\right)\right) .
\end{aligned}
$$

Using the estimates

$$
\begin{array}{r}
\left|e^{-(2 \pi n)^{2} \int_{0}^{t} a(s) d s}-e^{-(2 \pi n)^{2} \int_{0}^{t} b(s) d s}\right| \\
\leq(2 \pi n)^{2} T \max _{0 \leq t \leq T}|a(t)-b(t)|, \\
\left|e e^{-(2 \pi n)^{2} \int_{\tau}^{t} a(s) d s}-e^{-(2 \pi n)^{2} \int_{\tau}^{t} b(s) d s}\right| \\
\leq(2 \pi n)^{2} T \max _{0 \leq t \leq T}|a(t)-b(t)|,
\end{array}
$$

we obtain

$$
\max _{0 \leq t \leq T}|P[a(t)]-P[b(t)]| \leq \alpha \max _{0 \leq t \leq T}|a(t)-b(t)| .
$$

Let $\alpha \in(0,1)$ be arbitrary fixed number. Fix a number $T_{0}$, $0<T_{0} \leq T$, such that

$$
\frac{C_{1}\left(C_{4}+C_{5}\right)}{C_{2}^{2}} T_{0} \leq \alpha
$$

Then from the equality (10), we obtain

$$
\|a-b\|_{C\left[0, T_{0}\right]} \leq \alpha\|a-b\|_{C\left[0, T_{0}\right]},
$$

which implies that $a=b$. By substituting $a=b$ in (9), we have $u=v$.

\section{Continuous Dependence of $(a, u)$ on the Data}

Theorem 3. Under assumptions $\left(A_{1}\right)-\left(A_{3}\right)$, the solution $(a, u)$ of the problem (2)-(5) depends continuously on the data for small $T$.

Proof. Let $\Phi=\{\varphi, F, E\}$ and $\bar{\Phi}=\{\bar{\varphi}, \bar{F}, \bar{E}\}$ be two sets of the data, which satisfy the assumptions $\left(A_{1}\right)-\left(A_{3}\right)$. Then there exist positive constants $M_{i}, i=1,2,3$ such that

$$
\begin{gathered}
\|\varphi\|_{C^{4}[0,1]} \leq M_{1}, \\
\|F\|_{C^{4,0}\left(\bar{D}_{T}\right)} \leq M_{2}, \\
\|E\|_{C^{1}[0, T]} \leq M_{3}, \\
\|\bar{\varphi}\|_{C^{4}[0,1]} \leq M_{1}, \\
\|\bar{F}\|_{C^{4,0}\left(\bar{D}_{T}\right)} \leq M_{2}, \\
\|\bar{E}\|_{C^{1}[0, T]} \leq M_{3} .
\end{gathered}
$$

Let $(a, u)$ and $(\bar{a}, \bar{u})$ be solutions of the inverse problem (2)-(5) corresponding to the data $\Phi$ and $\bar{\Phi}$, respectively. According to (8),

$a(t)$

$$
=\frac{E^{\prime}(t)+\sum_{n=1}^{\infty}(1 / 2 \pi n) F_{n}(t)}{\sum_{n=1}^{\infty} 2 \pi n\left(\varphi_{n} e^{-(2 \pi n)^{2} \int_{0}^{t} a(s) d s}+\int_{0}^{t} F_{n}(\tau) e^{-(2 \pi n)^{2} \int_{\tau}^{t} a(s) d s} d \tau\right)},
$$

$\bar{a}(t)$

$$
=\frac{\bar{E}^{\prime}(t)+\sum_{n=1}^{\infty}(1 / 2 \pi n) \bar{F}_{n}(t)}{\sum_{n=1}^{\infty} 2 \pi n\left(\bar{\varphi}_{n} e^{-(2 \pi n)^{2} \int_{0}^{t} \bar{a}(s) d s}+\int_{0}^{t} \bar{F}_{n}(\tau) e^{-(2 \pi n)^{2} \int_{\tau}^{t} \bar{a}(s) d s} d \tau\right)} .
$$


First let us estimate the difference $a-\bar{a}$. It is easy to compute that

$$
\begin{aligned}
& \mid E^{\prime}(t) \sum_{n=1}^{\infty} 2 \pi n \bar{\varphi}_{n} e^{-(2 \pi n)^{2} \int_{0}^{t} \bar{a}(s) d s} \\
& -\bar{E}^{\prime}(t) \sum_{n=1}^{\infty} 2 \pi n \varphi_{n} e^{-(2 \pi n)^{2} \int_{0}^{t} a(s) d s} \\
& \leq M_{4}\|E-\bar{E}\|_{C^{1}[0, T]}+M_{5}\|\varphi-\bar{\varphi}\|_{C^{4}[0,1]} \\
& +M_{6}\|a-\bar{a}\|_{C[0, T]} \text {, } \\
& \mid E^{\prime}(t) \sum_{n=1}^{\infty} 2 \pi n \int_{0}^{t} \bar{F}_{n}(\tau) e^{-(2 \pi n)^{2} \int_{\tau}^{t} \bar{a}(s) d s} d \tau \\
& -\bar{E}^{\prime}(t) \sum_{n=1}^{\infty} 2 \pi n \int_{0}^{t} F_{n}(\tau) e^{-(2 \pi n)^{2} \int_{\tau}^{t} a(s) d s} d \tau \\
& \leq M_{7} T\|E-\bar{E}\|_{C^{1}[0, T]}+M_{5} T\|F-\bar{F}\|_{C^{4,0}\left(\overline{D_{T}}\right)} \\
& +M_{8}\|a-\bar{a}\|_{C[0, T]}, \\
& \mid \sum_{n=1}^{\infty} \frac{1}{2 \pi n} F_{n}(t) \sum_{n=1}^{\infty} 2 \pi n \bar{\varphi}_{n} e^{-(2 \pi n)^{2} \int_{0}^{t} \bar{a}(s) d s} \\
& -\sum_{n=1}^{\infty} \frac{1}{2 \pi n} \bar{F}_{n}(t) \sum_{n=1}^{\infty} 2 \pi n \varphi_{n} e^{-(2 \pi n)^{2} \int_{0}^{t} a(s) d s} \\
& \leq 2 \sqrt{6} M_{4}\|F-\bar{F}\|_{C^{4,0}\left(\overline{D_{T}}\right)}+2 \sqrt{6} M_{7}\|\varphi-\bar{\varphi}\|_{C^{4}[0,1]} \\
& +M_{9}\|a-\bar{a}\|_{C[0, T]}, \\
& \mid \sum_{n=1}^{\infty} \frac{1}{2 \pi n} F_{n}(t) \sum_{n=1}^{\infty} 2 \pi n \int_{0}^{t} \bar{F}_{n}(\tau) e^{-(2 \pi n)^{2} \int_{\tau}^{t} \bar{a}(s) d s} \\
& -\sum_{n=1}^{\infty} \frac{1}{2 \pi n} \bar{F}_{n}(t) \sum_{n=1}^{\infty} 2 \pi n \int_{0}^{t} F_{n}(\tau) e^{-(2 \pi n)^{2} \int_{\tau}^{t} a(s) d s} d \tau \\
& \leq \sqrt{6} T M\|F-\bar{F}\|_{C^{4,0}\left(\overline{D_{T}}\right)}+M_{10}\|a-\bar{a}\|_{C[0, T]},
\end{aligned}
$$

where $M_{k}, k=4, \ldots, 10$, are some constants.

If we consider these estimates in $a-\bar{a}$, we obtain

$$
\begin{aligned}
& \left(1-M_{11}\right)\|a-\bar{a}\|_{C[0, T]} \\
& \quad \leq M_{12}\left(\|E-\bar{E}\|_{C^{1}[0, T]}+\|\varphi-\bar{\varphi}\|_{C^{4}[0,1]}+\|F-\bar{F}\|_{C^{4,0}\left(\overline{D_{T}}\right)}\right) .
\end{aligned}
$$

The inequality $M_{11}<1$ holds for small $T$. Finally, we obtain

$$
\|a-\bar{a}\|_{C[0, T]} \leq M_{13}\|\Phi-\bar{\Phi}\|, \quad M_{13}=\frac{M_{12}}{\left(1-M_{11}\right)},
$$

where $\|\Phi-\bar{\Phi}\|=\|E-\bar{E}\|_{C^{1}[0, T]}+\|\varphi-\bar{\varphi}\|_{C^{4}[0,1]}+$ $\|F-\bar{F}\|_{C^{4,0}\left(\overline{D_{T}}\right)}$.
From (6), a similar estimate is also obtained for the difference $u-\bar{u}$ as

$$
\|u-\bar{u}\|_{C\left(\overline{D_{T}}\right)} \leq M_{14}\|\Phi-\bar{\Phi}\| .
$$

\section{Numerical Method}

We use the finite difference method with a predictor-corrector-type approach, that is suggested in [2]. Apply this method to the problem (2)-(5).

We subdivide the intervals $[0,1]$ and $[0, T]$ into $N_{x}$ and $N_{t}$ subintervals of equal lengths $h=\left(1 / N_{x}\right)$ and $\tau=\left(T / N_{t}\right)$, respectively. Then we add two lines $x=0$ and $x=\left(N_{x}+1\right) h$ to generate the fictitious points needed for dealing with the second boundary condition. We choose the Crank-Nicolson scheme, which is absolutely stable and has a second-order accuracy in both $h$ and $\tau$ [15]. The Crank-Nicolson scheme for (2)-(5) is as follows:

$$
\begin{aligned}
& \frac{1}{\tau}\left(u_{i}^{j+1}-u_{i}^{j}\right) \\
& =\frac{1}{2}\left(a^{j+1}+a^{j}\right) \frac{1}{2 h^{2}} \\
& \times\left[\left(u_{i-1}^{j}-2 u_{i}^{j}+u_{i+1}^{j}\right)+\left(u_{i-1}^{j+1}-2 u_{i}^{j+1}+u_{i+1}^{j+1}\right)\right] \\
& +\frac{1}{2}\left(F_{i}^{j+1}+F_{i}^{j}\right), \\
& u_{i}^{0}=\phi_{i}, \\
& u_{0}^{j}=u_{N_{x}}^{j} \\
& u_{1}^{j}=u_{N_{x}+1}^{j}
\end{aligned}
$$

where $1 \leq i \leq N_{x}$ and $0 \leq j \leq N_{t}$ are the indices for the spatial and time steps, respectively, $u_{i}^{j}=u\left(x_{i}, t_{j}\right), \phi_{i}=\varphi\left(x_{i}\right)$, $F_{i}^{j}=F\left(x_{i}, t_{j}\right)$, and $x_{i}=i h, t_{j}=j \tau$. At the $t=0$ level, adjustment should be made according to the initial condition and the compatibility requirements.

Equation (27) form an $N_{x} \times N_{x}$ linear system of equations

$$
A U^{j+1}=b,
$$

where

$$
U^{j}=\left(u_{1}^{j}, u_{2}^{j}, \ldots, u_{N_{x}}^{j}\right)^{\operatorname{tr}}, \quad 1 \leq j \leq N_{t},
$$

$$
b=\left(b_{1}, b_{2}, \ldots, b_{N_{x}}\right)^{\operatorname{tr}}
$$

$A=\left[\begin{array}{cccccccc}-2(1+R) & 1 & 0 & \cdots & & 0 & 1 \\ 1 & -2(1+R) & 1 & 0 & \cdots & & & 0 \\ 0 & 1 & -2(1+R) & 1 & 0 & \cdots & & 0 \\ \vdots & & & \ddots & & & & \\ & & & & & & & \\ 1 & & & & 0 & 1 & -2(1+R) & 1 \\ & & & & & 0 & 1 & -2(1+R)\end{array}\right]$, 


$$
\begin{gathered}
R=\frac{2 h^{2}}{\tau\left(a^{j+1}+a^{j}\right)}, \quad j=0,1, \ldots, N_{t}, \\
b_{1}=2(1-R) u_{1}^{j}-u_{2}^{j}-u_{N_{x}}^{j}-R \tau\left(F_{1}^{j+1}+F_{1}^{j}\right), \\
j=0,1, \ldots, N_{t}, \\
b_{N_{x}}=-u_{N_{x}-1}^{j}+2(1-R) u_{N_{x}}^{j}-u_{1}^{j} \\
-R \tau\left(F_{N_{x}}^{j+1}+F_{N_{x}}^{j}\right), \quad j=0,1, \ldots, N_{t}, \\
b_{i}=-u_{i-1}^{j}+2(1-R) u_{i}^{j}-u_{i+1}^{j}-R \tau\left(F_{i}^{j+1}+F_{i}^{j}\right), \\
\quad i=2,3, \ldots, N_{x}-1, \quad j=0,1, \ldots, N_{t} .
\end{gathered}
$$

Now, let us construct the predicting-correcting mechanism. First, multiplying (2) by $x$ from 0 to 1 and using (4) and (5), we obtain

$$
a(t)=\frac{E^{\prime}(t)-\int_{0}^{1} x F(x, t) d x}{u_{x}(1, t)} .
$$

The finite difference approximation of $(30)$ is

$$
a^{j}=\frac{\left[\left(\left(E^{j+1}-E^{j}\right) / \tau\right)-(\text { Fin })^{j}\right] h}{u_{N_{x}+1}^{j}-u_{N_{x}}^{j}},
$$

where $E^{j}=E\left(t_{j}\right),(\text { Fin })^{j}=\int_{0}^{1} x F\left(x, t_{j}\right) d x, j=0,1, \ldots, N_{t}$. For $j=0$,

$$
a^{0}=\frac{\left[\left(\left(E^{1}-E^{0}\right) / \tau\right)-(\text { Fin })^{0}\right] h}{\phi_{N_{x}+1}-\phi_{N_{x}}},
$$

and the values of $\phi_{i}$ help us to start our computation. We denote the values of $a^{j}, u_{i}^{j}$ at the sth iteration step $a^{j(s)}, u_{i}^{j(s)}$, respectively. In numerical computation, since the time step is very small, we can take $a^{j+1(0)}=a^{j}, u_{i}^{j+1(0)}=u_{i}^{j}, j=$ $0,1,2, \ldots N_{t}, i=1,2, \ldots, N_{x}$. At each $(s+1)$ th iteration step, we first determine $a^{j+1(s+1)}$ from the formula

$$
a^{j+1(s+1)}=\frac{\left[\left(\left(E^{j+2}-E^{j+1}\right) / \tau\right)-(\text { Fin })^{j+1}\right] h}{u_{N_{x}+1}^{j+1(s)}-u_{N_{x}}^{j+1(s)}} .
$$

Then from (27) we obtain

$$
\begin{aligned}
& \frac{1}{\tau}\left(u_{i}^{j+1(s+1)}-u_{i}^{j+1(s)}\right) \\
& =\frac{1}{4 h^{2}}\left(a^{j+1(s+1)}+a^{j+1(s)}\right) \\
& \quad \times\left[\left(u_{i-1}^{j+1(s+1)}-2 u_{i}^{j+1(s+1)}+u_{i+1}^{j+1(s+1)}\right)\right. \\
& \left.\quad+\left(u_{i-1}^{j+1(s)}-2 u_{i}^{j+1(s)}+u_{i+1}^{j+1(s)}\right)\right] \\
& +\frac{1}{2}\left(F_{i}^{j+1}+F_{i}^{j}\right), \\
& u_{0}^{j+1(s)}=u_{N_{x}}^{j+1(s)}, \\
& u_{1}^{j+1(s)}=u_{N_{x}+1}^{j+1(s)}, \quad s=0,1,2, \ldots
\end{aligned}
$$

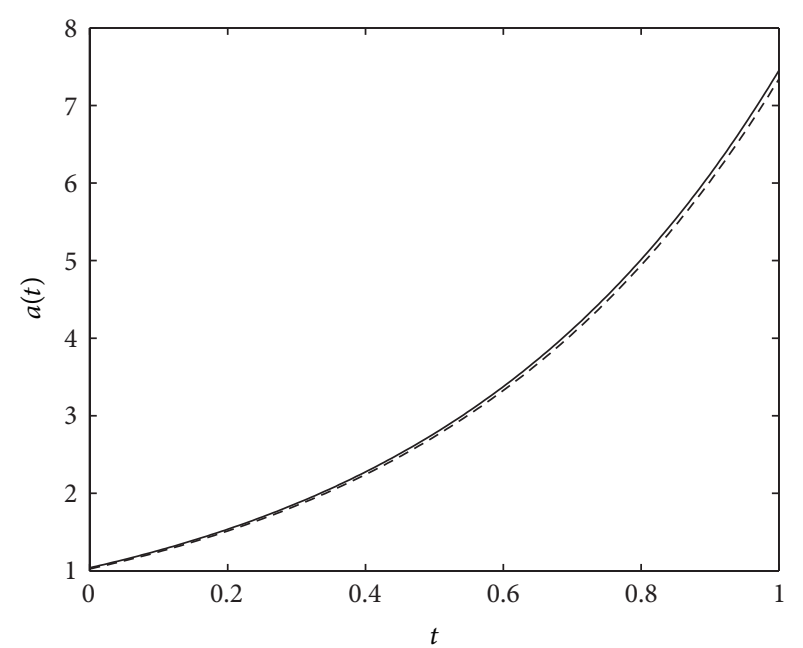

FIGURE 1: The analytical and numerical solutions of $a(t)$ when $T=1$. The analytical solution is shown with dashed line.

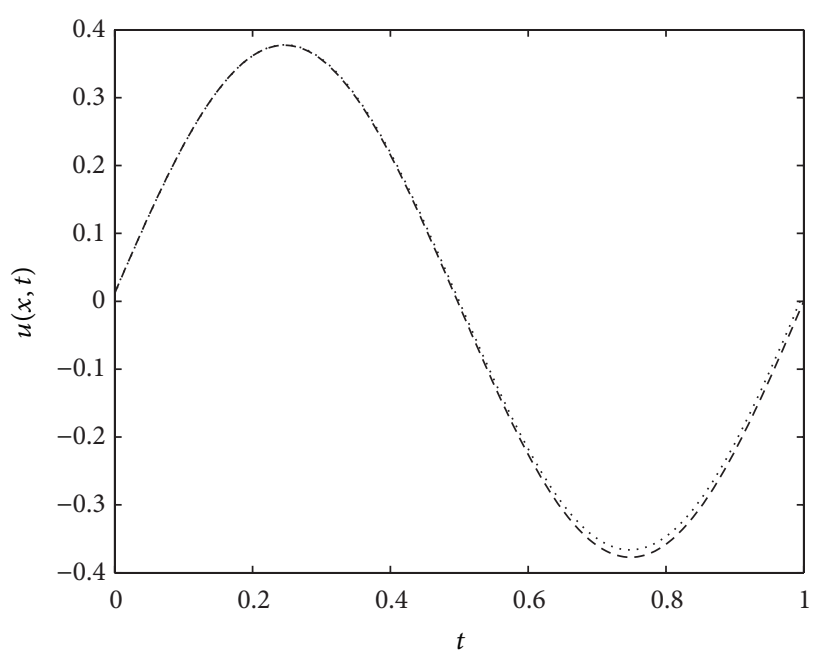

FIgURE 2: The analytical and numerical solutions of $u(x, t)$ at the $T=1$. The analytical solution is shown with dashed line.

The system of (34) can be solved by the Gauss elimination method and $u_{i}^{j+1(s+1)}$ is determined. If the difference of values between two iterations reaches the prescribed tolerance, the iteration is stopped and we accept the corresponding values $a^{j+1(s+1)}, u_{i}^{j+1(s+1)}\left(i=1,2, \ldots, N_{x}\right)$ as $a^{j+1}, u_{i}^{j+1}(i=$ $\left.1,2, \ldots, N_{x}\right)$, on the $(j+1)$ th time step, respectively. In virtue of this iteration, we can move from level $j$ to level $j+1$.

\section{Numerical Examples and Discussions}

Example 1. Consider the inverse problem (2)-(5), with

$$
\begin{gathered}
F(x, t)=(2 \pi)^{2} \sin (2 \pi x) \exp (t), \\
\varphi(x)=\sin (2 \pi x), \\
E(t)=-\frac{1}{2 \pi} \exp (-t), \\
x \in[0,1], t \in[0, T] .
\end{gathered}
$$




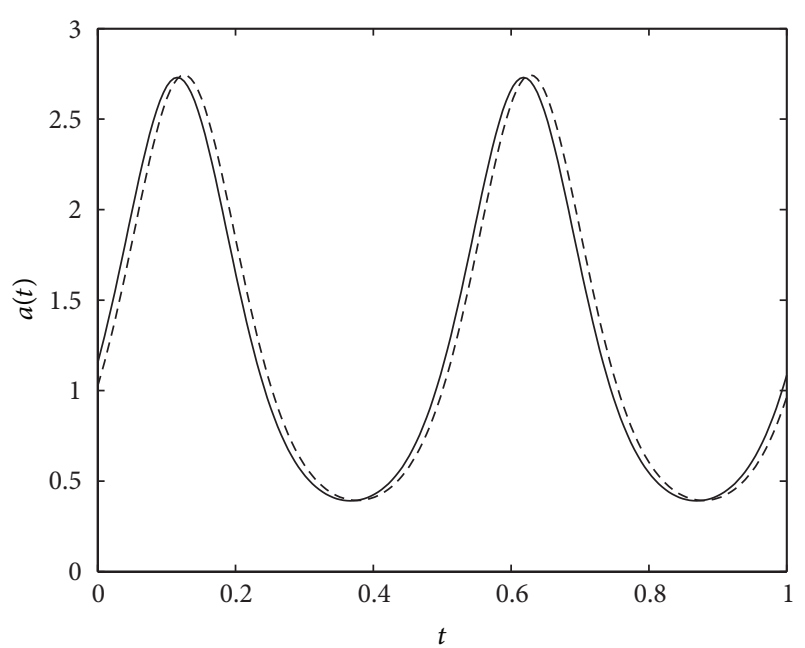

FIGURE 3: The analytical and numerical solutions of $a(t)$ when $T=1$. The analytical solution is shown with dashed line.

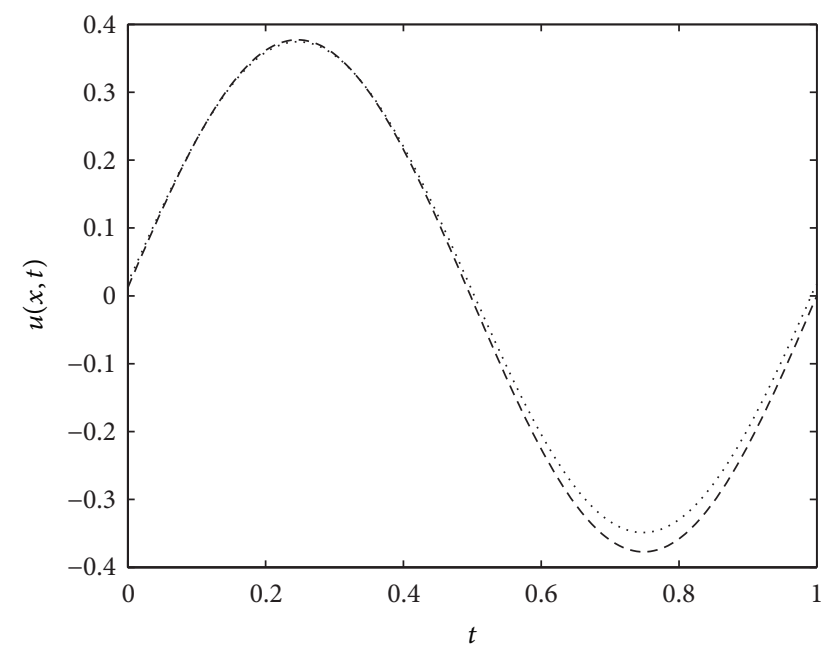

FIgURE 4: The analytical and numerical solutions of $u(x, t)$ at the $T=1$. The analytical solution is shown with dashed line.

It is easy to check that the analytical solution of the problem (2)-(5) is

$$
\{a(t), u(x, t)\}=\left\{\frac{1}{(2 \pi)^{2}}+\exp (2 t), \sin (2 \pi x) \exp (-t)\right\} .
$$

Let us apply the scheme which was explained in the previous section for the step sizes $h=0.005, \tau=0.005$.

In the case when $T=1$, the comparisons between the analytical solution (36) and the numerical finite difference solution are shown in Figures 1 and 2.
Example 2. Consider the inverse problem (2)-(5), with

$$
\begin{gathered}
F(x, t)=(2 \pi)^{2} \sin (2 \pi x) \exp (-t+\sin (4 \pi t)), \\
\varphi(x)=\sin (2 \pi x), \\
E(t)=-\frac{1}{2 \pi} \exp (-t), \\
x \in[0,1], t \in[0, T] .
\end{gathered}
$$

It is easy to check that the analytical solution of the problem (2)-(5) is

$$
\begin{aligned}
& \{a(t), u(x, t)\} \\
& \quad=\left\{\frac{1}{(2 \pi)^{2}}+\exp (\sin (4 \pi t)), \sin (2 \pi x) \exp (-t)\right\} .
\end{aligned}
$$

Let us apply the scheme which was explained in the previous section for the step sizes $h=0.01, \tau=h / 8$.

In the case when $T=1$, the comparisons between the analytical solution (38) and the numerical finite difference solution are shown in Figures 3 and 4.

\section{Conclusions}

The inverse problem regarding the simultaneously identification of the time-dependent thermal diffusivity and the temperature distribution in one-dimensional heat equation with periodic boundary and integral overdetermination conditions has been considered. This inverse problem has been investigated from both theoretical and numerical points of view. In the theoretical part of the paper, the conditions for the existence, uniqueness, and continuous dependence on the data of the problem have been established. In the numerical part, the sensitivity of the Crank-Nicolson finitedifference scheme combined with an iteration method with the examples has been illustrated.

\section{References}

[1] J. R. Cannon, Y. P. Lin, and S. Wang, "Determination of a control parameter in a parabolic partial differential equation," The Journal of the Australian Mathematical Society B, vol. 33, no. 2, pp. 149-163, 1991.

[2] J. R. Cannon, Y. Lin, and S. Wang, "Determination of source parameter in parabolic equations," Meccanica, vol. 27, no. 2, pp. 85-94, 1992.

[3] A. G. Fatullayev, N. Gasilov, and I. Yusubov, "Simultaneous determination of unknown coefficients in a parabolic equation," Applicable Analysis, vol. 87, no. 10-11, pp. 1167-1177, 2008.

[4] M. I. Ivanchov, "Inverse problems for the heat-conduction equation with nonlocal boundary condition," Ukrainian Mathematical Journal, vol. 45, no. 8, pp. 1186-1192, 1993.

[5] M. I. Ivanchov and N. V. Pabyrivska, "Simultaneous determination of two coefficients in a parabolic equation in the case of nonlocal and integral conditions," Ukrainian Mathematical Journal, vol. 53, no. 5, pp. 674-684, 2001.

[6] M. I. Ismailov and F. Kanca, "An inverse coefficient problem for a parabolic equation in the case of nonlocal boundary and overdetermination conditions," Mathematical Methods in the Applied Sciences, vol. 34, no. 6, pp. 692-702, 2011. 
[7] F. Kanca and M. I. Ismailov, "The inverse problem of finding the time-dependent diffusion coefficient of the heat equation from integral overdetermination data," Inverse Problems in Science and Engineering, vol. 20, no. 4, pp. 463-476, 2012.

[8] J. R. Cannon, "The solution of the heat equation subject to the specification of energy," Quarterly of Applied Mathematics, vol. 21, pp. 155-160, 1963.

[9] L. I. Kamynin, "A boundary value problem in the theory of heat conduction with a nonclassical boundary condition," Zhurnal Vychislitel'noi Matematiki i Matematicheskoi Fiziki, vol. 4, no. 6, pp. 1006-1024, 1964.

[10] N. I. Ionkin, "Solution of a boundary-value problem in heat conduction with a nonclassical boundary condition," Differential Equations, vol. 13, pp. 204-211, 1977.

[11] N. I. Yurchuk, "Mixed problem with an integral condition for certain parabolic equations," Differential Equations, vol. 22, pp. 1457-1463, 1986.

[12] V. M. Vīgak, "Construction of a solution of the heat conduction problem with integral conditions," Doklady Akademii Nauk Ukrainy, vol. 8, pp. 57-60, 1994.

[13] N. I. Ivanchov, "Boundary value problems for a parabolic equation with integral conditions," Differential Equations, vol. 40, no. 4, pp. 591-609, 2004.

[14] W. Liao, M. Dehghan, and A. Mohebbi, "Direct numerical method for an inverse problem of a parabolic partial differential equation," Journal of Computational and Applied Mathematics, vol. 232, no. 2, pp. 351-360, 2009.

[15] I. Sakinc, "Numerical solution of a quasilinear parabolic problem with periodic boundary condition," Hacettepe Journal of Mathematics and Statistics, vol. 39, no. 2, pp. 183-189, 2010.

[16] J. Choi, "Inverse problem for a parabolic equation with spaceperiodic boundary conditions by a Carleman estimate," Journal of Inverse and Ill-Posed Problems, vol. 11, no. 2, pp. 111-135, 2003. 


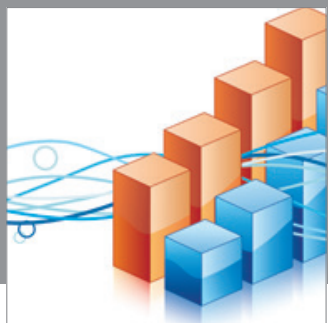

Advances in

Operations Research

mansans

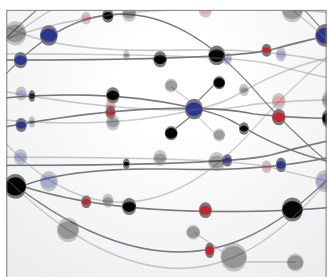

The Scientific World Journal
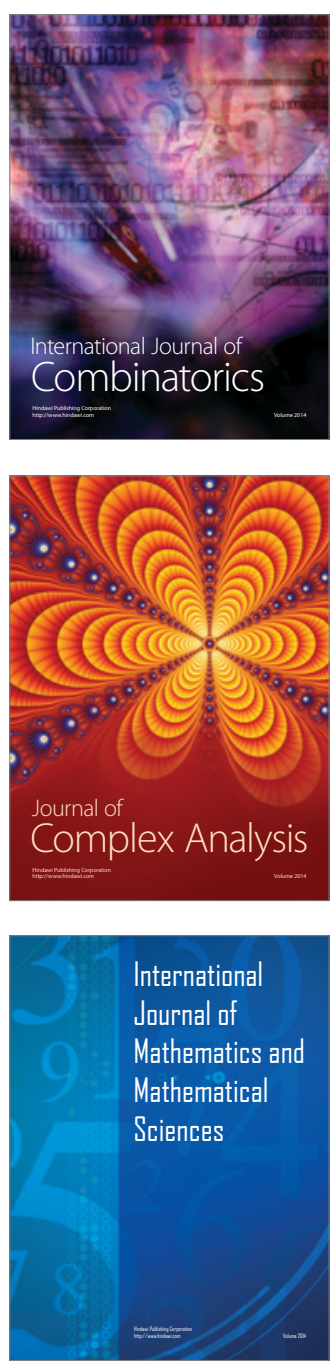
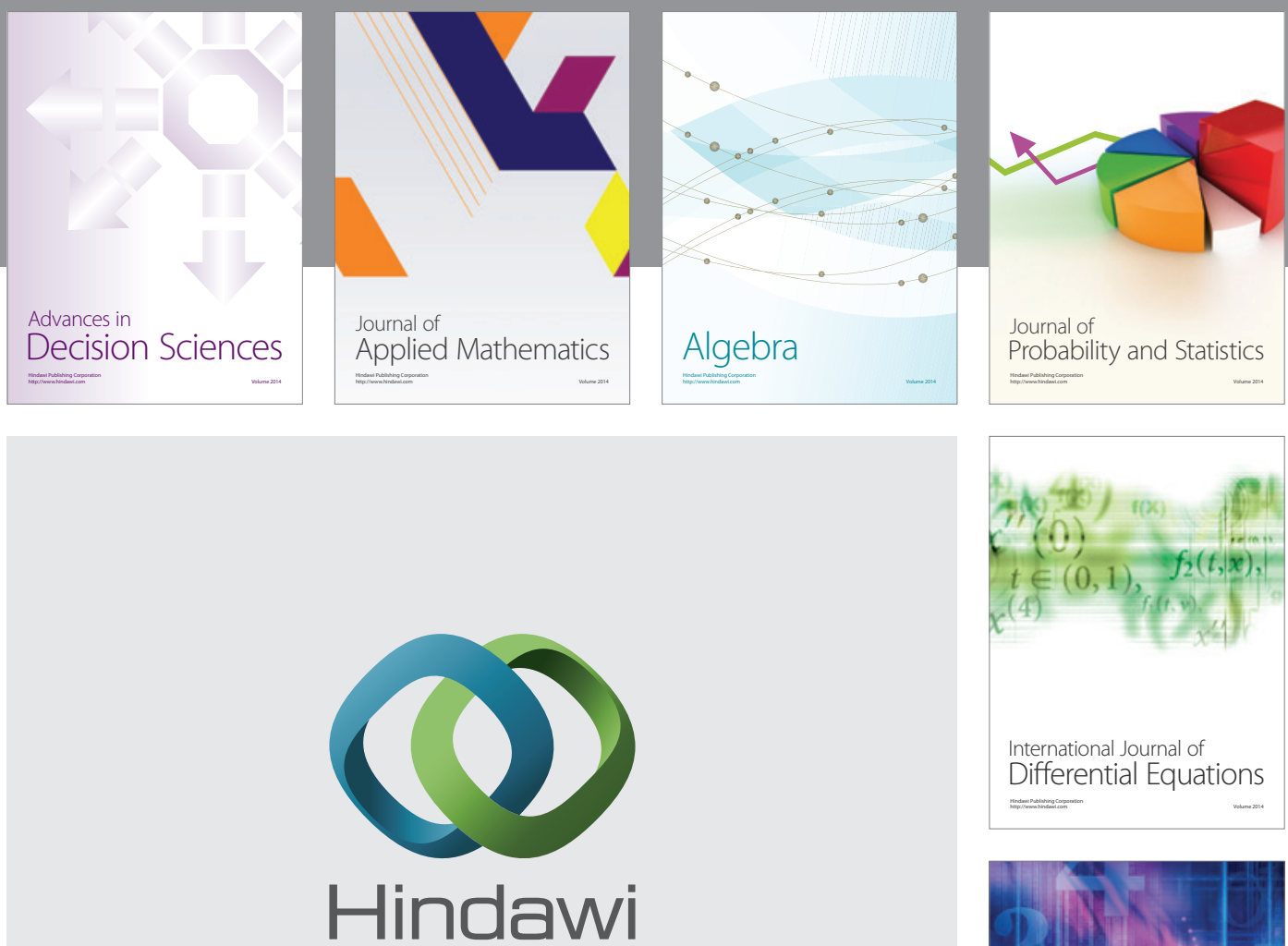

Submit your manuscripts at http://www.hindawi.com
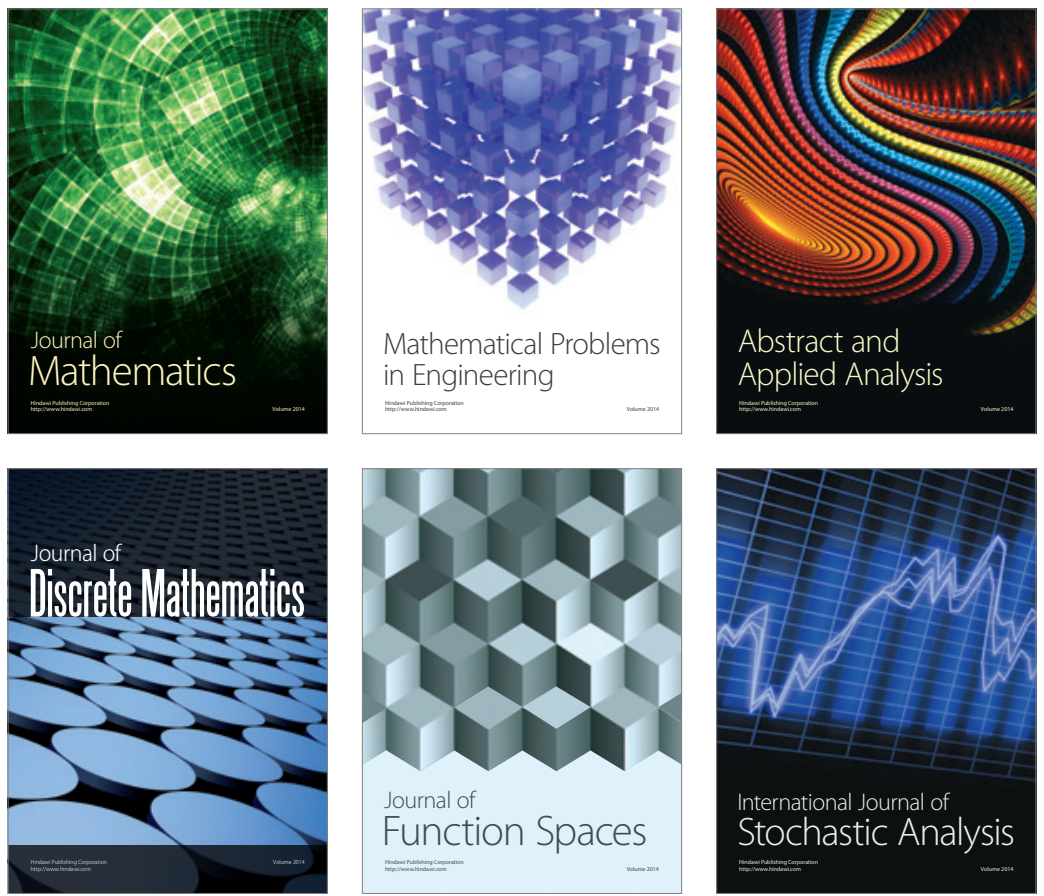

Journal of

Function Spaces

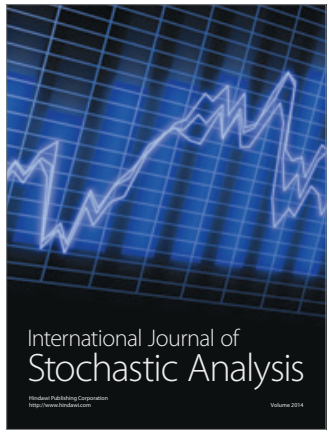

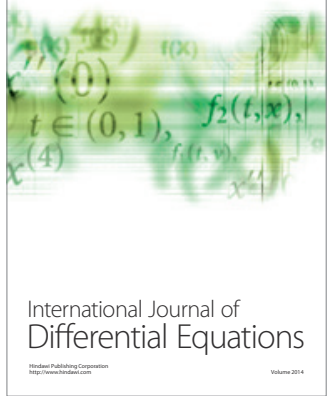
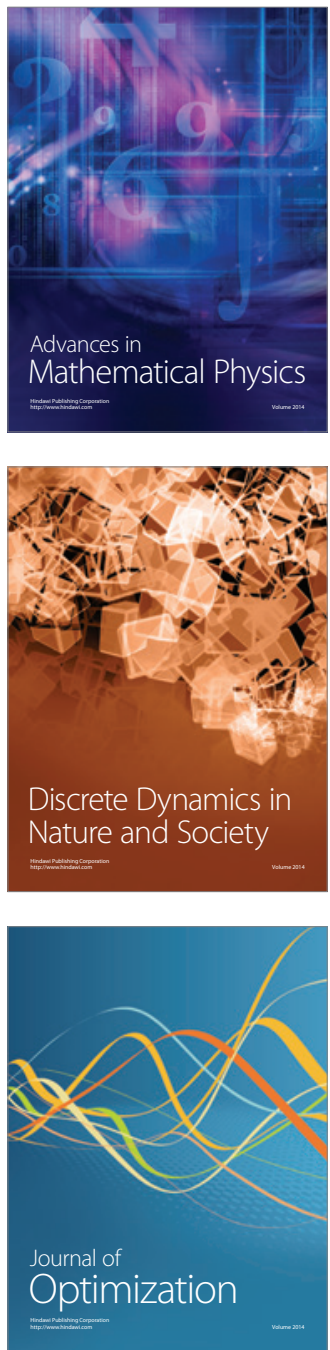